\title{
Some applications of $p$-summing operators \\ to Banach space theory
}

by

HASKELL P. ROSENTHAL (Urbana-Champaign, U.S.A.)

Abstract. Let $B$ be a Banach space. $B$ is said to be $p$-dominated if every operator from every $C(K)$-space to $B$ is $p$-absolutely summing. Equivalent formulations are developed in terms of certain finite dimensional inequalities in $B$. An exposition is given of the proof and some consequences of the Theorem: Every Banach space either contains $l_{n}^{\infty}$ 's or is $p$-dominated for some $2 \leqslant p<\infty$. (This result was first proved for quotient spaces of $O(K)$-spaces by the author and later shown in general by B. Maurey.) quotient spaces of $O(K)$-spaces by the author and later shown in general by B. Maurey.)
$\mathrm{A}$ new class of (finite-dimensional) bases is introduced, generalizing the class of "spreading" or "subsymmetric" bases. These bases are called unconditionally-transitive bases, It is shown that every such basis in a $p$-dominated space satisfies an upper$p$-function space estimate. This yields some fundamental inequalities of Tzafriri, used in his result that every space with unconditional basis contains uniformly complemented $l_{n}^{p}$ 's for some $p \in\{1,2, \infty\}$.

Our main objective is to give an exposition of the proof and consequences of the following fundamental result:

THEOREM 1. Let $B$ be an arbitrary Banach space. Then either $B$ contains $l_{n}^{\infty} s$ or there is a $2 \leqslant p<\infty$ so that every operator from every $C(K)$ space into $B$ factors through an $L^{p}(\mu)$-space.

(Our notation and definitions are standard for the most part, following the terminology of [17] and [27]. A Banach space is said to contain $l_{n}^{p}$ 's if there is a $\lambda$ so that it contains a $\lambda$-isomorph of $l_{n}^{p}$ for all $n$. "Operator" means "bounded linear operator"; a " $C(K)$-space" refers to the Banach space of real-valued continuous functions on some compact Hausdorff space $K$; an $L^{p}(\mu)$-space refers to the Banach space of equivalence classes of $p$ th-power integrable functions defined on some measure-space $(X, \mathscr{S} \mu)$; $L^{p}=L^{p}(\mu)$ for $\mu$ Lebesgue measure on the Lebesgue-measurable subsets of $[0,1]$. An operator $T: C(K) \rightarrow B$ factors through $L^{p}(\mu)$ if there are operators $U: C(K) \rightarrow L^{p}(\mu)$ and $V: L^{p}(\mu) \rightarrow B$ with $T=V U$.)

It is fairly easy to see that Theorem 1 has the following dual version: either a given Banach space $X$ is such that $X$ contains uniformly complemented $l_{n}^{1}$ 's, or there is a $1<q \leqslant 2$ so that every operator from $X$ to an $L^{1}(\mu)$ space factors through $L^{q}(\mu)$. For a striking recent application, see [11]. 
Theorem 1 was established in [26] for the case of quotient-spaces $B$ of $C(K)$-spaces. It was subsequently realized by Maurey [18] that the techniques in [26] actually yield the general case. The above-mentioned special case yields that every reflexive quotient space of a $C(K)$-space is isomorphic to a quotient space of an $L^{p}(\mu)$-space for some $2 \leqslant p<\infty$. By duality, one obtains the equivalent assertion that every reflexive subspace of an $L^{1}(\mu)$-space is isomorphic to a subspace of an $L^{p}(\mu)$-space for some $p>1$. We refer the reader to [26] and also the expository paper [27] for further applications of Theorem 1 and the more refined version presented in Section 2 below, to the structure of Banach subspaces of $L^{1}$, and also to [18], [20], and [21] for applications to the structure of Banach subspaces of the measurable functions. We shall be mainly concerned here with applications to the structure of general Banach spaces.

We develop our exposition of Theorem 1 in terms of the notion of $p$-absolutely summing operators. In Section 1 , we define this notion and summarize some of the elementary results. We also review some of the structure results of [26] concerning subspaces of $L^{1}$. We then define a Banach space $B$ to be $p$-dominated if every operator from every $C(K)$ space to $B$ is $p$-absolutely summing. Theorem 1 is implied by (and implies)

THEOREM 1'. Every Banach space either contains $l_{n}^{\infty} s$ or is p-domin" ated for some $2 \leqslant p<\infty$.

(We note in passing that the two possibilities of Theorem $I^{\prime}$ and Theorem 1 are easily seen to be mutually exclusive.)

The remainder of Section 1 is devoted to applications of Theorem $1^{\prime}$. These mainly are derivations of a number of inequalities discovered fairly recently. For example, it is shown in Corollary 1.3 that every unconditional basic sequence in a $p$-dominated space satisfies a lower-p-estimate, thus yielding a result of Johnson's [9]; a stronger result, used recently by Figiel and Johnson [5], is stated in Proposition 1.4. In Proposition 1.5 we state a dual consequence of Theorem $1^{\prime}$; namely, that every Banach space $B$ either contains $l_{n}^{1}$ 's or has the property that all of the quotient spaces of $B^{*}$ are $p$-dominated for some $2 \leqslant p<\infty$. Consequently we obtain in Corollary 1.6 that if a Banach space $B$ does not contain $l_{n}^{1}, s$, then every unconditional basic sequence in $B$ satisfies an upper- $q$-estimate for some $1<q \leqslant 2$. We then sketch an (unpublished) argument of Figiel which shows that if $B$ does not contain $l_{n}^{1}$, s, neither does $L^{2}(B)$; this allows the deduction in Corollary 1.7 of a result of Pisier [21], expose $N^{\circ} 7$; if $B$ does not contain $l_{n}^{1}$ 's, then $B$ is type $q$ for some $1<q \leqslant 2$ ("type $q$ " is defined in Section 1). The final result of Section 1, Corollary 1.8, gives a somewhat different proof of the result of Maurey that every cotype-2 Banach space is 2-dominated. (A special case is the fundamental theorem of Grothendieck [8]; $L^{1}$ is 2 -dominated.)
It thus appears that the inequalities implicit in Theorem $1^{\prime}$ are more powerful than these various other inequalities; naturally the direct proofs of these other results are often simpler than the proof of Theorem $1^{\prime}$ itself (though not simpler than their derivation from $1^{\prime}$ ). In the interests of economy of thought and power of method, it seems worthwhile to realize these other inequalities as special cases of the one set of inequalities given by Theorem $1^{\prime}$.

We present the proof of Theorem $1^{\prime}$ in Section 2, via the notion of $p$-inclusive Banach spaces (defined at the beginning of Section 2). A stronger result is stated in Theorem 2.1, namely the result of Maurey [18]: for $2<p<\infty$, a Banach space is either $p$-dominated or $p$-inclusive, and these alternatives are mutually exclusive. (It is this theorem which generalizes the main result of [26].) A crucial step in the proof of Theorem 2.1 is a "variation-of-density lemma". A "motivated" proof is given in [27], and is consequently not repeated here. Theorem 2.1 also involves an application of a technique of Giesy [6] to $p$-inclusive Banach spaces (stated as Proposition 2.3).

In Section 3, we introduce a generalization of the class of subsymmetric or "spreading" bases, namely the class of unconditionally transitive bases. We then use this notion and Theorem $1^{\prime}$ to recover some fundamental inequalities of Tzafriri [29], called by us the Tzafriri functionspace estimates. These estimates are one of the key steps in his important result that every infinite dimensional Banach space with unconditional basis contains uniformly complemented $l_{n}^{p}$ 's for $p=1,2$, or $\infty$. It is a famous open question as to whether this remains true if the phrase "with unconditional basis" is deleted. For recent progress, see [11].

Our style is largely expository with "minimal background" required for most of the arguments. A fair amount of attention is given to stating recent related results. The reader already expert in these matters will probably find it most beneficial to focus on Section 3 and parts of Section 2 .

1. Applications of Theorem $\mathbf{1}^{\prime}$. We begin with some elementary considerations concerning $p$-summing operators. Let $1 \leqslant p<\infty$ and $X$ and $Y$ be Banach spaces. An operator $T: X \rightarrow Y$ is called $p$-absolutely summing ( $p$-a.s.) if for any sequence $\left(x_{j}\right)$ in $X$ for which $\Sigma\left|x^{*}\left(x_{j}\right)\right|^{p}<\infty$ for all $x^{*}$ in $X^{*}$, one has $\sum\left\|T x_{j}\right\|^{p}<\infty$. It is easily seen that this occurs if and only if there is a $\lambda<\infty$ so that for any finite sequence $x_{1}, \ldots, x_{n}$ in $X$,

$$
\left(\sum\left\|T x_{j}\right\|^{p}\right)^{1 / p} \leqslant \lambda \sup \left(\sum\left|x^{*}\left(x_{j}\right)\right|^{p}\right)^{1 / p},
$$

the supremum taken over all $x^{*}$ in $X^{*}$ of norm one. The smallest possible $\lambda$ satisfying this inequality is called the $p$-a.s. norm of $T$. It is a fairly simple consequence of the local reflexivity principle (as presented in [10]) that if $T$ is $p$-a.s. with $p$-a.s. norm $\lambda$, then also $T^{* *}$ is $p$-a.s. with $p$-a.s. norm $\lambda$. 
Now suppose that $X$ is a subspace of a. $O(K)$-space and $T: X \rightarrow Y$ is $p$-a.s. It is then easily seen, using the Riesz representation theorem, that the $p$-a.s. norm of $T$ is equal to the smallest $\lambda$ so that for all $x_{1}, \ldots, x_{n}$ in $X$,

$$
\left(\sum\left\|T x_{j}\right\|^{p}\right)^{1 / p} \leqslant \lambda \sup _{k \in \mathcal{K}}\left(\sum\left|x_{j}(k)\right|^{p}\right)^{1 / p} .
$$

A non-trivial equivalence is the following result due to Pietsch (see [23] and [15]):

Pietscir Factorization Theorem. Let $X, Y, C(K)$, and $T$ be as above. Then $T$ has $p$-a.s. norm at most $\lambda$ if and only if there is a probability Borel measure $\mu$ on $K$ so that

$$
\|T x\| \leqslant \lambda\left(\int_{K}|x(k)|^{p} d \mu(k)\right)^{1 / p} \quad \text { for all } x \in X .
$$

It follows immediately that if an operator is $p$-a.s., then it is $q$-a.s. for all $q>p$. Now suppose that $X=C(K)$, and $\mu$ and $T$ are as above. Since the natural map $i$ of $C(K)$ into $L^{p}(\mu)$ has dense range, one trivially obtains the existence of an operator $V: L^{p}(\mu) \rightarrow Y$ with $\|V\| \leqslant \lambda$ so that $T=V i$. Thus every $p$-summing operator on a $C(K)$-space factors through an $L^{p}(\mu)$-space. The converse is false for all $2<p<\infty$, as we shall see in Section 2 (this result is due to Schwartz-Kwapien: see [13]). However, a result of Kwapien's ([14], see also [26]) asserts that if an operator on a $C(K)$-space factors through an $L^{p}$-space $(2<p<\infty)$, then it is $q$-summing for all $p<q$.

The Pietsch Factorization Theorem evidently gives a global result using only the "data" of the local behavior of an operator, that is, its behavior on finite-dimensional subspaces. On the other hand, the results just mentioned have considerable content and applicability in the case where $T$ is defined on a finite-dimensional $C(K)$-space.

Propositron 1.1. Fix $n$ a positive integer and suppose $K=\{1,2, \ldots, n\}$, i.e., $C(K)=l_{n}^{\infty}$. Let $B$ be a Banach space and $T: C(K) \rightarrow B$ a given operator. Put $b_{i}=T e_{i}$ for all $i, 1 \leqslant i \leqslant n$, where $e_{i}(j)=\delta_{i j}$ for all $i$ and $j$; assume that $b_{i} \neq 0$ for all $i$.

(1) $\|T\|=\sup \left\|\sum_{i=1}^{n} \pm b_{i}\right\|$, the supremum taken over all choices of signs \pm 1 .

Let $1<p<\infty$. The $p$-a.s. norm of $T$ is the smallest number $\lambda$ satisfying any of the following four conditions:

(2) $\sum_{i}\left\|\sum_{j} x_{i}(j) b_{j}\right\|^{p} \leqslant \lambda^{p} \sup _{1 \leqslant j \leqslant n} \sum_{i}\left|x_{i}(j)\right|^{p}$ for any finite sequence $x_{1}, \ldots, x_{m}$ in $O(K)$.

(3) $\sum_{j}\left(\sum_{i}\left|y_{i}(j) b_{j}\right|^{p}\right)^{1 / p} \leqslant \lambda\left(\sum_{i}\left\|y_{i}\right\|^{p}\right)^{1 / p}$ for any finite sequence $y_{1}, \ldots, y_{m}$ in $B^{*}$
(4) There exist positive numbers $\mu_{i}, \ldots, \mu_{n}$ with $\sum \mu_{i}=1$ so that $\left\|\sum x(j) b_{j}\right\|$ $\leqslant \lambda\left(\left.\sum|x(j)|^{p} \mu_{j}\right|^{1 / p}\right.$ for all $x \in C(K)$.

(5) There exist positive numbers $\mu_{1}, \ldots, \mu_{n}$ with $\sum \mu_{i}=1$ so that $\left(\sum\left|b^{*}\left(b_{i}\right)\right|^{q} \mu_{i}^{q-1}\right)^{1 / q} \leqslant \lambda\left\|b^{*}\right\|$ for all $b^{*}{ }_{\epsilon} B^{*}$, where $1 / q+1 / p=1$.

Sketch of the proof. (We leave the details to the reader; all the equivalences follow simply from our previous considerations.) (1) and (2) follow from the definitions. (3) is equivalent to (2) by duality (cf. Proposition 1 of [26]). (2) $\Leftrightarrow(4)$ follows from the Pietsch Factorization Theorem. Finally, $(4) \Leftrightarrow(5)$ follows from duality: Let $\mu$ be the natural measure on $\{1, \ldots, n\}$ induced by $\mu_{1}, \ldots, \mu_{n}$, and define $S: L^{p}(\mu) \rightarrow B$ by $S(x)=\Sigma x(j) b_{j}$. (4) evidently asserts that $\|S\| \leqslant \lambda$, which is equivalent to $\mid S^{*} \| \leqslant \lambda$, where $\left(L^{p}(\mu)\right)^{*}$ is identified with $L^{q}(\mu)$. For $f \epsilon L^{p}(\mu)$ and $g \epsilon L^{p}(\mu)$, the natural pairing is given by $\langle f, g\rangle=\Sigma f(i) g(i) \mu_{i}$. It follows that for all $b^{*} \in B^{*}$, $S^{*} b^{*}(i)=b^{*}\left(b_{i}\right) / \mu_{i}$; thus (5) is simply the assertion that $\left\|S^{*}\right\| \leqslant \lambda$.

The next (preliminary) result "summarizes dual-characterizations of $p$-summing operators.

Proposition 1.2. Let $\mu$ be a probability measure on some measurable space, $X$ a Banach space, $1<p<\infty, 1 / p+1 / q=1, \lambda<\infty$, and $T: X \rightarrow L^{1}(\mu)$ a given operator. Then the following three statements are equivalent:

$1^{\circ}$ For any positive integer $n$ and elements $x_{1}, \ldots, x_{n}$ of $X$,

$$
\int\left(\sum\left|T x_{i}\right|^{p}\right)^{1 / p} d \mu \leqslant \lambda\left(\sum\left\|x_{i}\right\|^{p}\right)^{1 / p} .
$$

$2^{\circ} T^{*}: L^{\infty}(\mu) \rightarrow B^{*}$ has $q$-a.s. norm less than or equal to $\lambda$.

$3^{\circ}$ There exists a non-negative measurable function $\varphi$ with $\int \varphi d \mu \leqslant 1$ so that for all $x \in X,(T x)(t)=0$ for (almost) all $t$ belonging to $\{t: \varphi(t)=0\}$ and

$$
\left(\int|T x|^{p} \varphi^{1-p} d \mu\right)^{1 / p} \leqslant \lambda\|x\| .
$$

Implications $(1) \Leftrightarrow(3)$ as well as certain generalizations thereof, were demonstrated by Maurey in [18] using a minimax theorem. Theorem 1 of [26] yields 1.2 under the special case where $X$ is already a subspace of $L^{1}(\mu)$ and $T$ is the identity injection. Actually the proof given in [26] may be adapted almost verbation to obtain the above result. Assuming 2 , the function $p$ is produced by using the Pietsch Factorization Theorem and identifying $L^{\infty}(\mu)$ with a $C(K)$-space. The measure $\nu$ which arises from the Pietsch Factorization Theorem is then shown to be of the form $\varphi d \mu$, thanks to the Radon-Nikodym theorem.

In the case in which $X$ is already a subspace of $L^{1}(\mu)$ and $T$ is the identity injection, the smallest $\lambda$ satisfying $3^{\circ}$ is denoted by $I_{p}(X)$. As pointed out in [24], the condition " $I_{p}(X)<\infty$ " is not an isomorphic invariant of $X$, for $2<p$. However, it is an isomorphic invariant for $p \leqslant 2$; moreover, if $X$ and $Y$ are isometric subspaces of $L^{1}(\mu)$, then $I_{p}(X)$ 
$=I_{p}(Y)$. This is because of the fact that there exists a sequence $f_{1}, f_{2}, \ldots$ in $L^{1}$, isometrically equivalent to the usual basis of $l^{p}$. Consequently, a subspace $X$ of $L^{1}(\mu)$ satisfies $1^{\circ}$ of 1.2 for $T$ equal to the identity injection if and only if

$$
\int_{0}^{1}\left\|\sum f_{i}(\omega) x_{i}\right\| d \omega \leqslant \lambda\left(\sum\left\|x_{i}\right\|^{p}\right)^{1 / p}
$$

for all $x_{1}, \ldots, x_{n}$ in $X$. If we fix the $f_{i}^{\prime}$ 's to be independent identically distributed symmetric random variables, stable of exponent $p$, then the existence of such a $\lambda$ is an isomorphic invariant on $X$, definable regardless of whether or not $X$ imbeds in $L^{1}$. (A symmetric stable random variable of exponent $p$ defined on $[0,1]$, is by definition a measurable function $f$ on $[0,1]$ so that for some non-zero $c, \int_{0}^{1} e^{i t f(x)} d x=e^{-|c t|^{p}}$ for all real $t$. For the existence and properties of such functions, see most standard text on probability theory or [25].) Following Pisier [24], we say that a Banach space $X$ is type $p$-stable if there exists a $\lambda<\infty$ such that the above in equality holds for all $n$ and $x_{1}, \ldots, x_{n}$ in $X$. It is a fact (cf. [24] or Corollary 2.4 below) that $L^{s}$ is type $p$-stable for all $1 \leqslant p<s \leqslant 2$. If $X$ is type $p$-stable with constant $\lambda$, then one immediately obtains that $1^{\circ}$ of 1.2 holds for every norm-one operator $T: X \rightarrow L^{1}(\mu)$. This implies that every operator $S$ from a $C(K)$-space to $X^{*}$ is $q$-a.s., with $q$-a.s. norm at most $\|S\| \lambda$, where $1 / p+1 / q=1$. In particular, if $X$ is a subspace of $L^{1}(\mu)$ with $I_{p}(X)<\infty$, then every operator $S$ from a $C(K)$-space to $X^{*}$ is $q$-a.s. with $q$-a.s. norm at most $\|S\| I_{p}(X)$ (cf. [26]). By duality, one obtains that if there is a $q$-summing surjective map from a $C(K)$-space onto a Banach space $B$, then every map from any $O(K)$-space into $B$ is $q$-summing. Before passing to consequences of Theorem $1^{\prime}$, we note one final consequence of Proposition 1.2 (i.e. of Theorem 1 of [26]) itself: $I_{2}\left(l^{2}\right)=\sqrt{\pi / 2}$ (This identity is valid for real Banach spaces, and is due to Grothendieck [8].) For complex spaces, Grothendieck obtains that $I_{2}\left(l^{2}\right)=2 / \sqrt{\pi}$. (I am indebted to A. Pelczyński for bringing this to my attention.) The identity has also been obtained by Gordon and Lewis [7].) To see this, it suffices to take a particular subspace $X$ of $L^{1}$ isometric to $l^{2}$ Let $x_{1}, x_{2}, \ldots$ be a sequence of independent standard normal random variables and let $X$ equal the closed-linear-space of the $x_{j}$ 's in $L^{1}$. Then if $x$ is in $X, x \neq 0, x$ is also a normal random variable, hence $\left\|x_{2}\right\| /\|x\|_{1}$ $=\sqrt{\pi / 2}$; this shows that $I_{2}\left(l^{2}\right) \leqslant \sqrt{\pi / 2}$. To show the reverse inequality, because of $1^{\circ}$ of 1.2 and the facts that $\left\|x_{i}\right\|_{2}=1$ and $\left\|x_{i}\right\|_{1}=\sqrt{2 / \pi}$ for all $i$ it suffices to prove that

$$
\lim _{n \rightarrow \infty} n^{-\frac{1}{2}} \int_{0}^{1}\left(x_{1}^{2}(t)+\ldots+x_{n}^{2}(t)\right)^{\frac{1}{2}} d t=1 .
$$

Of course, this is a simple consequence of the strong law of large numbers.

Let $2<p<\infty$. We say that a Banach space $B$ is $p$-dominated if every operator from every $C(K)$-space into $B$ is $p$-a.s. (From now on, it is understood that " $p$-dominated" refers to some $2 \leqslant p<\infty$.) It is easily seen that if $B$ is $p$-dominated, then there is an $M<\infty$ so that every operator $T$ from every $C(K)$-space into $B$ has $p$-a.s. norm at most $M\|T\|$; if $M$ satisfies this relationship for all such operators $T$, we say that $B$ is $M$-p-dominated. Now as is well-known ([16]), a $C(K)$-space has the property that for each $\lambda>1$ and each of its finite dimensional subspaces $F$, there exists an $n$ and a subspace $G$ with $F \subset G$ and $G \lambda$-isomorphic to $l_{n}^{\infty}$. We then obtain, using Proposition 1.1, that a Banach space is $M$-p-dominated if and only if for any $n$ and $b_{1}, \ldots, b_{n}$ in $B$, the (equivalent) assertions (2)-(5) of 1.1 hold, where one sets $\lambda$ equal to $M \sup _{ \pm}\left\|\sum \pm b_{i}\right\|$. We use the word "dominated" in this definition because of the inequality which results from applying (4). This inequality shows that the sum of a certain series is dominated by an $L^{p}$ function-space estimate. We return to this observation in Section 3. We note in passing that one of our previous remarks asserts that Hilbert space is $\sqrt{\pi / 2}$-2-dominated and $\sqrt{\pi / 2}$ is the best possible constant. For a discussion of various properties of 2 -dominated Banach spaces, see [2]

Let $1 / p+1 / q=1$. It follows easily from the Persson-Pietsch duality theory [22] that a Banach space $B$ is $p$-dominated if and only if every $q$-a.s. operator from $B$ into an arbitrary Banach space is 1-a.s. The results in [18] are usually expressed in this alternate formulation. It should also be pointed out that a Banach space $B$ is $p$-dominated if and only if every operator from every (or from some) infinite dimensional $\mathscr{L}_{\infty}$-space to $B$ is $p$-a.s. (see [16] for the definition and fundamental properties of $\mathscr{L}_{\infty}$ spaces). Moreover, if $B^{*}$ is $p$-dominated, every operator from $B$ to $L^{1}$ factors through $L^{q}$, while if every operator from $B$ to $L^{1}$ factors through $L^{q}$, then $B^{*}$ is $r$-dominated for all $r>p$.

From now on, " $u$ " shall denote a real number greater than or equal to one. Say that a normalized sequence $\left(b_{j}\right)$ in a Banach space is $u$-unconditional if for all $n$, scalars $c_{1}, \ldots, c_{n}$ and choices of signs \pm

$$
\left\|\sum \pm c_{j} b_{j}\right\| \leqslant u\left\|\sum c_{j} b_{j}\right\| \text {. }
$$

Now it follows immediately from the definitions that if $B$ is $M$ - $p$-dominated, then every $u$-unconditional sequence $\left(x_{j}\right)$ in $B$ satisfies a lower$M u-p$ estimate; i.e., for all scalars $c_{1}, \ldots, c_{n}$

$$
\left(\sum\left|c_{i}\right|^{p}\right)^{1 / p} \leqslant M u\left\|\sum c_{j} x_{j}\right\| .
$$


To see this, one simply sets $b_{j}=c_{j} x_{j}$ for all $j ; x_{i}(j)=\delta_{i j}$, and applies (2) with

$$
\lambda=M \sup _{ \pm}\left\|\sum \pm c_{j} x_{j}\right\| \leqslant M u\left\|\sum c_{j} x_{j}\right\|
$$

Hence we have the following consequence of Theorem $1^{\prime}$.

Corollarr 1.3. If a Banach space $B$ does not contain $l_{n}^{\infty} s$, then there is a $2 \leqslant p<\infty$ so that every unconditional basic sequence in $B$ satisfies a lower-p-estimate.

Of course, a special case of Corollary 1.3 is that if a Banach space does not contain $l_{n}^{\infty}$ 's and has an unconditional basis, then this basis must be $p$-block Besselian for some $2 \leqslant p<\infty$; this result was established directly by $\mathrm{W}$. Johnson in [9].

Actually, a considerably stronger inequality than the above can be proved; this stronger inequality is formulated and used in a recent paper of Figiel and Johnson [5]; the case $p=2$ is discussed in [2].

Proposimion 1.4. Let a Banach space $B$ have a u-unconditional basis $u_{1}, u_{2}, \ldots$ and let $B$ be $M$ - $p$-dominated. Then for any $n$ and sequence $y_{1}, y_{2}, \ldots$ $\ldots, y_{n}$ in $B$,

$$
\left(\sum|| y_{i} \|^{p}\right)^{1 / p} \leqslant M u\left\|\sum_{i}\left(\sum_{j}\left|y_{j}(i)\right|^{p}\right)^{1 / p} u_{i}\right\|,
$$

where $y_{j}=\sum y_{j}(i) u_{i}$ for all $j$.

To see this, assume that $y_{j} \neq 0$ for any $j$ and put $d_{i}=\left(\sum_{j}\left|y_{j}(i)\right|^{p}\right)^{1 / p}$ for all $i$ (by our remarks preceding $1.3, d_{i}<\infty$ for all $i$ ). Now set $x_{i}(j)$ $=\frac{y_{i}(j)}{d_{j}}$ for all $i$ and $j$. Then $\sum_{i}\left|x_{i}(j)\right|^{p}=1$ for all $j$. Hence the inequality of 1.4 follows immediately from (2) upon setting $b_{i}=\left(\sum_{j}\left|y_{j}^{\mathrm{z}}(i)\right|^{p}\right)^{1 / p} u_{i}$ for all $i$ and $\lambda=M \sup \left\|\sum \pm b_{i}\right\| \leqslant M u\left\|\sum b_{i}\right\|$. We note also that the results of [2] imply that the converse of 1.4 holds if $p=2$; that is, if $B$ has an unconditional basis and satisfies the conclusion of 1.4, then $B$ is 2 -dominated.

We now give some dual consequences of Theorem 1 .

Proposimion 1.5. A Banach space $B$ either contains $l_{n}^{1}$ s, or there exists an $M$ and $a p$ so that every quotient space of $B^{*}$ is $M$-p-dominated.

Proof. Suppose that the second alternative fails to hold and fix $n$. The proof of Theorem $1^{\prime}$ given in Section 2 yields that there is an $M$ and a $p$ so that if a Banach space $X$ fails to be $M$ - $p$-dominated, then it contains a 2-isomorph of $l_{n}^{\infty}$. Of course, such a 2 -isomorph is automatically 2 -complemented; thus $X$ has a quotient space 4 -isomorphic to $l_{n}^{\infty}$. Oonsequently, $B^{*}$ has a quotient space 4 -isomorphic to $l_{n}^{\infty}$, so $B^{* *}$ has a subspace 4 -isomorphic to $l_{n}^{1}$. Hence by the local reflexivity principle, $B$ contains $l_{n}^{1}$ ss.

Now it is an immediate consequence of the remarks directly preceding 1.3 that if a Banach space $B$ is such that every quotient space of $B$ is $M$-p-dominated, then every $u$-unconditional sequence $\left(x_{j}\right)$ in $B$ satisfies an upper- $M u$ - $q$-estimate, where $1 / p+1 / q=1$; i.e., for all $n$ and scalars $c_{1}, \ldots, c_{n}$,

$$
\left\|\sum c_{j} x_{j}\right\| \leqslant M u\left(\sum\left|c_{j}\right|^{q}\right)^{1 / q} .
$$

We thus obtain the following consequence of 1.5 :

CoROLLARY 1.6. If a Banach space $B$ does not contain $l_{n}^{1}$ 's, then there is a $1<q \leqslant 2$ so that every unconditional basic sequence in $B$ satisfies an upper-q-estimate.

Let $1<q \leqslant 2$ and $r_{1}, r_{2}, \ldots$ denote the Rademacher functions defined on $[0,1]$. (Of course, $r_{1}, r_{2}, \ldots$ is just a particular representation of a sequence of $\{1,-1\}$-valued symmetric independent random variables; we leave out the Rademacher function $r_{0}=1$.) Following Pisier [24], a Banach space $B$ is said to be of type- $q$ if there is a constant $K$ so that for all $n$ and $b_{1}, \ldots, b_{n}$ in $B$,

$$
\frac{1}{2^{n}} \sum_{ \pm}\left(\left\|\sum_{i} \pm b_{i}\right\|^{\|^{\alpha}}\right)^{1 / \alpha}=\left(\int\left\|\sum_{i} r_{i}(t) b_{i}\right\|^{q} d t\right)^{1 / \alpha} \leqslant K\left(\sum_{i}\left\|b_{i}\right\|^{\alpha}\right)^{1 / \alpha} .
$$

(The first equality is trivial.) (A remarkable result of Kahane's [12] asserts that for all $0<q, r<\infty$, there exist constants $K_{q, r}$ so that for any Banach space $B$ and $b_{1}, \ldots, b_{n}$ in $B$,

$$
\left(\int\left\|\sum r_{i}(t) b_{i}\right\|^{q} d t\right)^{1 / q} \leqslant K_{q, r}\left(\int\left\|\sum r_{i}(t) b_{i}\right\|^{r}\right)^{1 / r} .
$$

This generalizes the classical Khintchine inequalities which constitute the above assertion with "for any Banach space $B$ " replaced by " $B$ equal to the set of scalars".)

The notion of "type-2" seems to have been first introduced in [2] as "subquadratic Rademacher average". It is proved in [2] that if $B$ " is type-2, then $B$ is 2 -dominated. We present a stronger result due to Maurey in Corollary 1.8 .

Our final application of 1.5 is the following result due to Pisier [19], exposé No. 7 :

CoRollary 1.7. If a Banach space $B$ does not contain $l_{n}^{1}$ 's, then it is of type- $q$ for some $1<q \leqslant 2$.

Proof. It is a fact that if $B$ does not contain $l_{n}^{1}$ 's, then neither does $L^{2}(B)$. (We sketch a proof due to Figiel, momentarily.) Consequently, there is an $M$ and a $1<q \leqslant 2$ so that every 1-unconditional sequence 
in $L^{2}(B)$ satisfies an upper-M-q-estimate, by 1.6. But if we fix $n$ and let $b_{1}, \ldots, b_{n}$ be non-zero elements of $B$, then the sequence $r_{1}(t) \frac{b_{1}}{\left\|b_{1}\right\|}, \ldots$ $\ldots, r_{n}(t) \frac{b_{n}}{\left\|b_{n}\right\|}$ may be considered as a 1-unconditional sequence in $L^{2}(B)$ This proves 1.7. The fact is seen as follows: A result of Giesy [6] (discussed in Section 2) yields that if a Banach space $X$ contains $l_{n}^{1}$ 's, then it contains $\lambda$-isomorphs of $l_{n}^{1}$ for all $\lambda>1$ and all $n$. In turn, the latter condition is easily seen to be equivalent to the existence for each $n$ and $\lambda>1$ of $n$ elements $b_{1}, \ldots, b_{n}$ in $B$ of norm-one so that $n \leqslant \lambda\left\|\sum \pm b_{i}\right\|$ for all choices of signs \pm . Now suppose that $L^{2}(B)$ contains a subspace isometric to $l_{n}^{1}$. Then we could choose vectors $x_{1}(t), \ldots, x_{n}(t)$ in $L^{2}(B)$, each of norm one, so that for all choices of signs, $\pm n=\left(\int\left\|\sum_{i} \pm x_{i}(t)\right\|^{2} d t\right)^{1 / 2}$ But for each $t,\left\|\sum \pm x_{i}(t)\right\| \leqslant \sum\left\|x_{i}(t)\right\|$; of course, then $\left(\int \sum\left\|x_{i}(t)\right\|^{2} d t\right)^{1 / 2}=n$. Consequently, $\left\|\sum \pm x_{i}(t)\right\|=\sum\left\|x_{i}(t)\right\|$ a.e.; thus this equality must also hold a.e. for all choices of signs. Since we have that $\left(\int \sum\left\|x_{i}(t)\right\|^{2} d t\right)^{1 / 2}=\sum\left(\int\left\|x_{i}(t)\right\|^{2}\right)^{1 / 2}$ $=n$, it follows from the strict convexity of $L^{2}$ that $\left\|x_{i}(t)\right\|=\left\|x_{1}(t)\right\|$ a.e. for all $i$. We thus have, after simply picking a $t$ for which the above relations hold and $\left\|x_{1}(t)\right\| \neq 0$, that

$$
\left\|\sum \pm \frac{x_{i}(t)}{\left\|x_{i}(t)\right\|}\right\|=\frac{\left\|\sum \pm x_{i}(t)\right\|}{\left\|x_{1}(t)\right\|}=\frac{\sum\left\|x_{i}(t)\right\|}{\left\|x_{1}(t)\right\|}=n
$$

for all choices of signs \pm ; hence $\left(\frac{x_{i}(t)}{\left\|x_{i}(t)\right\|}\right)_{i=1}^{n}$ is isometrically equivalent to the usual basis of $l_{n}^{1}$. Now thanks to the uniform convexity of $L^{2}$, this argument goes through in the approximate setting as well. Given $\varepsilon>0$, there exists a $\delta>0$ (depending on $n$ only) so that if $n-\delta \leqslant\left(\int\left\|\sum \pm x_{i}(t)\right\|^{2} d t\right)^{1 / 2}$ for all choices of signs \pm (with each $x_{i}$ of norm 1), then there exists a set $E$ of positive measure so that for all $t \epsilon E,\left\|x_{1}(t)\right\|>0$, $\left|\left\|x_{j}(t)\right\|-\left\|x_{1}(t)\right\|\right|<\varepsilon\left\|x_{1}(t)\right\|$ for all $j$, and $\left\|\sum \pm x_{i}(t)\right\| \geqslant(1-\varepsilon) \sum\left\|x_{i}(t)\right\|$ for all choices of signs \pm . It follows that $\left\|x_{j}(t)\right\| \neq 0$ for all $j$ and $\left\|\sum \frac{ \pm x_{i}(t)}{\left\|x_{i}(t)\right\|}\right\|$ $\geqslant\left((1-\varepsilon)^{2}-\varepsilon\right) n$ for all choices of signs \pm . Given $\lambda>1$, then if $\varepsilon$ is sufficiently small, we have that $\left(\frac{x_{i}(t)}{\left\|x_{i}(t)\right\|}\right)_{i=1}^{n}$ is equivalent to the usual $l_{n}^{1}$-basis, hence if $L^{2}(B)$ contains $l_{n}^{1}$ s, so does $B$.

The proof of 1.7 given by Pisier is direct; this enabled him to give an alternate proof of the result of [26] that reflexive subspaces of $L^{1}$ imbed in $L^{\alpha}$ for some $q>1$ (of course, this also provides another proof of the fact mentioned above, as well as 1.6). In subsequent joint work Maurey and Pisier showed (see [21], exposé No. 8 that for an arbitrary
Banach space $B$, the set of $q<2$ such that $B$ is type $q$-stable, is an open interval. This yjelds an alternate proof of Theorem 8 of [26].

A Banach space $B$ is said to be of cotype-2 if there is a $K$ so that for all $n$ and $y_{1}, \ldots, y_{n}$ in $B$,

$$
\left(\sum\left\|y_{i}\right\|^{2}\right) \leqslant K \int_{0}^{1}\left\|\sum r_{i}(t) y_{i}\right\| d t
$$

where $r_{1}, \ldots, r_{n}, \ldots$ denote the Rademacher functions. The classical Khintchine inequalities show that $L^{1}$ is cotype-2. Hence the final result of this section (due to Maurey) generalizes the theorem of Grothendieck that $L^{1}$ is 2 -dominated.

Since $B^{*}$ of type 2 implies $B$ is of type 2 , it also generalizes the result of [2] mentioned before 1.7. Our proof of this result is somewhat different than Maurey's.

COROLLARY 1.8 (Maurey [18]). Every cotype-2 Banach space $B$ is 2-dominated.

Pro of. Since $B$ is of cotype-2, it follows that $B$ does not contain $l_{n}^{\infty} / \mathrm{s}$ hence by Theorem 1 there is a $2 \leqslant p<\infty$ so that $B$ is $p$-dominated. Now let $K$ be chosen as in the definition of cotype-2. To complete the proof, it suffices to show that there is a constant $A$ so that for every operator $T$ from a $C(K)$-space into $B$ such that $T$ has $p$-a.s. norm-one, $T$ has 2 -a.s. norm at most $A$. We shall show that $A$ can be taken equal to $K \cdot K_{p, 2}$, where $K_{p, 2}$ is the constant arising in the classical Khintchine inequalities (i.e., the constant defined following 1.6 for the scalars-case). Since it suffices to consider only $C(S)$-spaces for $S$ finite, by $(2)$ of 1.1 it suffices to prove that given $n$ and non-zero $b_{1}, \ldots, b_{n}$ in $B$ satisfying

$$
\sum_{i}\left\|\sum_{j} x_{i}(j) b_{j}\right\|^{p} \leqslant \sup _{1 \leqslant j \leqslant n} \sum_{i}\left|x_{i}(j)\right|^{p}
$$

for all $m$ and $x_{1}, \ldots, x_{m}$ in $l_{m}^{\infty}$, then

$$
\sum_{i}\left\|\sum_{j} x_{i}(j) b_{j}\right\|^{2} \leqslant A^{2} \sup _{1 \leqslant j \leqslant n} \sum_{i}\left|x_{i}(j)\right|^{2}
$$

for all $m$ and $x_{1}, \ldots, x_{m}$ in $l_{m}^{\infty}$.

So, fix non-zero $b_{1}, \ldots, b_{n}$ in $B$ satisfying (a). Then choose $\mu_{1}, \ldots, \mu_{n}$ as in (4) of 1.1, satisfying the inequality of (4) (for $\lambda=1$ ); then fix $m$ and $x_{1}, \ldots, x_{m}$ in $l_{m}^{\infty}$. We then have

$$
\left(\sum_{i}\left\|\sum_{j} x_{i}(j) b_{j}\right\|^{2}\right)^{1 / 2} \leqslant K \int\left\|\sum_{j} \sum_{i} r_{i}(t) x_{i}(j) b_{j}\right\| d t
$$

(since $B$ is cotype-2)

$$
\leqslant K \int\left(\sum_{j}\left|\sum_{i} r_{i}(t) x_{i}(j)\right|^{p} \mu_{j}\right)^{1 / p} d t
$$


(by applying (4) for each fixed $t$, where $x(j)=\sum_{i} r_{i}(t) x_{i}(j)$ )

$$
\leqslant K\left(\sum_{j} \mu_{j}\left(\int\left|\sum r_{i}(t) x_{i}(j)\right|^{p} d t\right)^{1 / p}\right)
$$

(by Hölder's inequality)

$$
\leqslant K K_{p, 2}\left(\sum_{j} \mu_{j}\left(\sum_{i}\left|x_{i}(j)\right|^{2}\right)^{p / 2}\right)^{1 / p}
$$

(by Khintchine's inequalities)

$$
\leqslant K K_{p, 2} \sup _{1 \leqslant j \leqslant n}\left(\sum_{i}\left|\infty_{i}(j)\right|^{2}\right)^{1 / 2}
$$

(since $\sum \mu_{j}=1$ and $\mu_{j} \geqslant 0$ for all $j$ ). Thus (b) holds, completing the proof. (For further properties of spaces of cotype-2, see [18]).

2. $p$-dominated and $\dot{p}$-inclusive Banach spaces. A fundamental dichotomy. Let $n$ be a positive integer, $\lambda \geqslant 1$, and $1 \leqslant p<2$ or $2<p \leqslant \infty$ be given We say that a Banach space is $n$ - $\lambda$-p-inclusive if it contains $n$ norm- 1 elements $b_{1}, \ldots, b_{n}$ so that for all scalars $c_{1}, \ldots, c_{n}$,

$$
\begin{array}{ccc}
\left\|\sum c_{i} b_{i}\right\| \leqslant \lambda\left(\sum\left|c_{i}\right|^{p}\right)^{1 / p} \quad \text { if } \quad 2<p \leqslant \infty ; \\
\left(\sum\left|c_{i}\right|^{p}\right)^{1 / p} \leqslant \lambda\left\|\sum c_{i} b_{i}\right\| \quad \text { if } \quad 1 \leqslant p<2 .
\end{array}
$$

We say that a Banach space is $p$-inclusive if there is a $\lambda \geqslant 1$ so that it is $n$ - $\lambda$-p-inclusive for all positive integers $n$. We have left out the case $p=2$, since the famous Dvoretzky theorem [3] asserts that every infinite-dimensional Banach space contains $\lambda$-isomorphs of $l_{n}^{2}$ for all $n$ and $\lambda>1$; hence we cannot use the $p=2$ case as a distinguishing isomorphic invariant. Of course, a Banach space is 1-inclusive if and only if it contains $l_{n}^{1}$ ss. We shall see below that if a Banach space is $p$-inclusive, then it is in fact $n-\lambda-p$-inclusive for all $n$ and $\lambda>1$. This implies that a Banach space is $\infty$-inclusive if and only if it contains $\eta_{n}^{\infty}$,s. (Indeed, suppose $1 \leqslant \lambda<2$ and the norm one elements $b_{1}, \ldots, b_{n}$ in $B$ satisfy $(6)$ for $p=\infty$ and all scalars $c_{1}, \ldots, c_{n}$. Then for any $b^{*}$ in $B^{*}, \sum\left|b^{*}\left(b_{j}\right)\right| \leqslant \lambda\left\|b^{*}\right\|$. Now for each $i$ let $b_{i}^{*}$ in $B^{*}$ be such that $\left\|b_{i}^{*}\right\|=1=b_{i}^{*}\left(b_{i}\right)$. Then $\sum_{j} b_{i}^{*}\left(b_{j}\right)$ $\leqslant \lambda-1$. Fix $i$ and suppose $c_{1}, \ldots, c_{n}$ are given scalars with $\left|c_{i}\right|=\sup \left|c_{j}\right|$. Then $\left|b_{i}^{*}\left(\sum c_{j} b_{j}\right)\right| \geqslant\left|c_{i}\right|(2-\lambda)$, consequently the span of $\left\{b_{1}, \ldots, b_{n}\right\}$ is $\frac{\lambda}{2-\lambda}$-isomorphic to $l_{n}^{\infty}$.) It also implies that, if a Banach space is $q$ inclusive for all $q$ with $2<q<p \leqslant \infty$ (resp. $1 \leqslant p<q<2$ ), then it is $p$-inclusive. To see this, note that for $n$ and $p$ fixed, $\lim _{\alpha \rightarrow p}\left(\sum_{i=1}^{n}\left|c_{i}\right|^{\alpha}\right)^{1 / \alpha}=\left(\sum_{i=1}^{n}\left|c_{i}\right|^{p}\right)^{1 / p}$ for $p<\infty\left(\right.$ resp. $=\sup \left|o_{i}\right|$ for $\left.p=\infty\right)$ uniformly over the set of $c_{1}, \ldots, c_{n}$ with $\sup \left|c_{i}\right| \leqslant 1$. Phrased another way, the set of $p$ such that a Banach space is $p$-inclusive forms a closed subinterval of $[1,2)$ or of $(2, \infty]$. We shall also see that if a Banach space is $p$-inclusive for some $2<p<\infty$, then its dual is $q$-inclusive, where $1 / q+1 / p=1$. The converse is trivially false, for $L^{1}$ is not $p$-inclusive for any $2<p \leqslant \infty$, yet $L^{\infty}$ is $q$-inclusive for all $q$.

Maurey's generalization of the main result of [26] may now be phrased as follows:

Trenorem 2.1. Let $2<p<\infty$ and $B$ be an arbitrary Banach space. Then $B$ is $p$-dominated or $p$-inclusive. These alternatives are mutually exclusive.

Theorem $I^{\prime}$ follows immediately from Theorem 2.1 and our above remarks. Indeed, if a space is not $p$-dominated for any $p<\infty$, it is $p$-inclusive for all $p<\infty$, hence also $\infty$-inclusive; thus the space contains $l_{n}^{\infty}$ ss. It also follows (as pointed out jn [18]) that the set of $p>2$ for which a Banach space is $p$-dominated, forms an open subinterval of $(2, \infty)$, simply because the set of $p>2$ satisfying the other alternative is of the form $(2, r]$, if non-empty.

We pass now to the proof of Theorem 2.1. The first assertion follows immediately from the following quantitative result:

THEOREM 2.2. Let $2<q<\infty, n$ a positive integer, and $\lambda>1$ be given. Then there exists an $M$ so that if a Banach space $B$ is not $M$-q-dominated, it is $n-\lambda$-q-inclusive.

Proof. Let $M$ be large, the correct size to be decided later. Assuming $B$ is not $M$-q-dominated, there exists a finite set $K$ and an operator $S$ : $C(K) \rightarrow B$ of norm one so that $N>M$, where $N$ equals the $q$-a.s. norm of $S$. Since $K$ is finite, $N<\infty$. By the Pietsch Factorization Theorem, there exists a probability measure $\mu$ on $K$ so that setting $X=B^{*}$ and regarding $T=S^{*}$ as having its range in $L^{1}(\mu)$, then $T$ is a norm-one operator with the following properties:

$$
\begin{aligned}
& N=\sup _{\|x\| \leqslant 1}\|T x\|_{L_{(\mu)}^{p}} ; \\
& N \leqslant \sup _{\|x\| \leqslant 1}\|T x\|_{L_{(p d \mu)}^{p}}
\end{aligned}
$$

for all positive $\mu$-probability densities $p$, where $1 / p+1 / q=1$.

(See (5) of 1.1 which leads to 3 of Proposition 1.2 in this setting.) At this point, we shall simply regard $\mu$ as a probability measure on some measurable space $(\Omega, \mathscr{P})$.

Now let $0<\delta<1$ be a number close to 1 , depending on $n, p$ and $\lambda$. (One can define $\delta$ by $\lambda=\left(1-n^{1 / q} 2\left(1-\delta^{p}\right)^{1 / p}\right)^{-1}$.) We then have 
LEMCMA 2.3. If $M$ is large enough, there exist $x_{1}, \ldots, x_{n}$ in $X$ of normone and disjoint measurable sets $E_{1}, \ldots, E_{n}$ so that

$$
N \delta \leqslant\left(\int_{E_{i}}\left|T x_{i}\right|^{p} d \mu\right)^{1 / p} \quad \text { for all } i .
$$

the span of $\left(u_{i j}\right)_{j=1}^{n}$ so that

$$
b_{i}=\sum_{j=1}^{n} b_{i j} w_{i j} \quad \text { with } \quad\left(\sum_{j=1}^{n}\left|b_{i j}\right|^{p}\right)^{1 / p} \leqslant \frac{1}{\sqrt{\lambda}} .
$$

But then for any scalars $c_{1}, \ldots, c_{n}$,

This result is stated and proved in Section 3 of [27], it is called there a "variation-of-density lemma". (See also the proof of the Sublemma, p. 362 of [26].) We deduce 2.2 as follows: the vectors $\left(\chi_{Z_{i}} T x_{i}\right)_{i=1}^{n}$ span a subspace of $L^{p}(\mu)$ isometric to $l_{n}^{p}$, which is the range of a contractive projection. It follows by a standard perturbation argument that the span, of the vectors $\left(T x_{i}\right)_{i=1}^{n}$ almost has these properties. (Only the closeness of the $T x_{i}$ 's to the usual $l_{n}^{p}$-basis was used in [26]; the use of the existence of a nice projection onto their span is due to Maurey.) Precisely, one may let $\tau$ be the canonical isometry from the linear span of the $\chi_{E_{i}} T x_{i}$ 's onto $l_{n}^{p}$ such that $\tau\left(\chi_{E} T x_{i}\right)=\left\|\chi_{E_{i}} T x_{i}\right\|_{L^{p_{(\mu)}}} e_{i}$ for all $i$, where $\left(e_{i}, \ldots, e_{n}\right)$ is the usual $l_{n}^{p}$-basis. Let $P$ be the canonical contractive projection from $L^{p}(\mu)$ onto the linear span of the $\chi_{E_{i}} T x_{i}$ 's and let $Q: l_{n}^{p} \rightarrow l_{n}^{p}$ be such that $Q \tau P\left(T x_{i}\right)=e_{i}$ for all $i$. It can then be shown that $\|Q\| \leqslant \lambda N^{-1}$, where $\delta$ is defined as above; consequently the map $U=Q \tau P T$ from $X$ to $l_{n}^{p}$ has the property that $\|U\| \leqslant \lambda$ and $U x_{i}=e_{i}$ for all $i$. Returning to our original setting, the map $U$ is also weak*-continuous; that is, there is an operator $V: l_{n}^{q} \rightarrow B$ so that $U=V^{*}$. Then $\|V\| \leqslant \lambda$ too; the fact that $\left\|x_{i}\right\|=1$ and $V^{*} x_{i}=e_{i}$ implies that $\left\|V \tilde{e}_{i}\right\| \geqslant 1$ for all $i$, where $\left(\tilde{e}_{1}, \ldots, \tilde{e}_{n}\right)$ is the usual basis for $l_{n}^{q}$. It follows that putting $b_{i}=V \tilde{e}_{i} /\left\|V \tilde{e}_{i}\right\|$ for all $i$, then

$$
\left\|\sum c_{j} b_{j}\right\| \leqslant \lambda\left(\sum_{i}\left|c_{j}\right|^{q} /\left\|\nabla \tilde{e}_{j}\right\|^{q}\right)^{1 / \alpha} \leqslant \lambda\left(\sum\left|c_{j}\right|^{q}\right)^{1 / q}
$$

for all scalars $c_{1}, \ldots, c_{n}$, thus completing the proof of 2.2 .

To prove the second assertion of Theorem 2.1 we shall use the following consequence of a result of Giesy. [6]:

Proposition 2.3. If a Banach space is $p$-inclusive, then it is $n-\lambda-p$ inclusive for all $\lambda>1$ and all positive integers $n$.

Proof. It is obviously enough to show that for a fixed $\lambda>1$ and a fixed positive integer $n$, if $B$ is $n^{2}-\lambda$-p-inclusive, then $B$ is $n-\sqrt{\lambda}-p$-inclusive. Let $2<p \leqslant \infty$ and $\left(u_{i j}\right)_{i, j=1}^{n}$ be norm-one elements in $B$ so that

$$
\left\|\sum_{i, j} c_{i j} u_{i j}\right\| \leqslant \lambda\left(\sum_{i, j}\left|c_{i j}\right|^{2}\right)^{1 / p}
$$

for all scalars $\left(c_{i j}\right)_{i, j=1}^{n}$ (we define $\left(\sum_{i, j}\left|c_{i j}\right|^{p}\right)^{1 / p}=\sup _{i, j}\left|c_{i j}\right|$ when $p=\infty$ ).

If there is an $i$ so that $\left\|\sum c_{j} u_{i j}\right\| \leqslant \sqrt{\lambda}\left(\sum\left|c_{j}\right|^{2}\right)^{1 / p}$ for all scalars $c_{1}, \ldots, c_{n}$, we are done. If not, we may choose for each $i$ a norm-one element $b_{i}$ in

$$
\begin{aligned}
\left\|\sum c_{i} b_{i}\right\| & =\left\|\sum_{i, j} c_{i} b_{i j} u_{i j}\right\| \\
& \leqslant \lambda\left(\sum_{i}\left(\left|c_{i}\right|^{p} \sum_{j}\left|b_{i j}\right|^{p}\right)\right)^{1 / p} \quad \text { by (10) } \\
& \leqslant \frac{\lambda}{\sqrt{\lambda}}\left(\sum\left|c_{i}\right|^{p}\right)^{1 / p} \text { by (11), }
\end{aligned}
$$

which proves the result for $p>2$. The proof for $1 \leqslant p<2$ is almost the some and shall be omitted.

Now suppose that $B$ is $p$-inclusive for some $2<p<\infty$, let $1 / p+$ $+1 / q=1$, and fix $n$ a positive integer and $\lambda>1$. It follows from 2.3 that there exist elements $b_{1}, \ldots, b_{n}$ in $B$ so that $\sup _{i}\left|c_{i}\right| \leqslant \| \sum_{i} c_{i} b_{i} \mid$ $\leqslant \lambda\left(\sum_{i}\left|c_{i}\right|^{p}\right)^{1 / p}$ for all scalars $c_{1}, \ldots, c_{n}$. To see this, let $\beta>1$ and let $x_{1}, \ldots, x_{n}$ be norm-one elements in $B$ so that $\left\|\sum c_{i} x_{i}\right\| \leqslant \beta\left(\sum\left|c_{i}\right|^{p}\right)^{1 / p}$ for all scalars $c_{1}, \ldots, c_{n}$. Then $\left(\sum\left|x^{*}\left(x_{i}\right)\right|^{q}\right)^{1 / \alpha} \leqslant \beta\left\|x^{*}\right\|$ for all $x^{*} \in B^{*}$. Fix $i$ and choose $x_{i}^{*}$ so that $x_{i}^{*}\left(x_{i}\right)=1=\left\|x_{i}^{*}\right\|$. Then

$$
\sum_{j \neq i}\left|x_{i}^{*}\left(x_{j}\right)\right| \leqslant\left(\beta^{q}-1\right)^{1 / q} n^{1 / p} .
$$

Assume thait $\left(\beta^{q}-1\right)^{1 / q} n^{1 / p}<1$. It follows that for any scalars $c_{1}, \ldots, c_{n}$,

$$
\left\|\sum c_{j} x_{j}\right\| \geqslant \sup \left|c_{j}\right|\left(1-\left(\beta^{q}-1\right)^{1 / q} n^{1 / p}\right) \text {. }
$$

If we put $b_{j}=\left(1-\left(\beta^{q}-1\right)^{1 / q} n^{1 / p}\right)^{-1} x_{j}$ for all $j$, then $b_{1}, \ldots, b_{n}$ have the desired properties provided $\beta\left(1-\left(\beta^{\alpha}-1\right)^{1 / \alpha} n^{1 / p}\right)^{-1} \leqslant \lambda$.

In turn, the existence of such $b_{f}$ 's and the Hahn-Banach theorem show that there exist an operator $T: B^{*} \rightarrow l_{n}^{q}$ with $\|T\| \leqslant \lambda$ and norm-one elements $b_{1}^{*}, \ldots, b_{n}^{*}$ in $B^{*}$ so that $T b_{i}^{*}=e_{i}$ for all $i$, where $\left(e_{1}, \ldots, e_{n}\right)$ is the standard basis of $l_{n}^{q}$

We àre finally prepared to follow the argument given by Maurey in [18] to complete the proof of Theorem 2.1. Suppose $B$ is also $M-p$ dominated for some $M<\infty$. Let $f_{1}, f_{2}, \ldots$ be identically distributed symmetric independent random variables, stable of exponent $q$, so that $\left\|f_{1}\right\|_{1}=1$. Thus the sequence $\left(f_{j}\right)$ is isometrically equivalent in the $L^{\mathbf{1}}$ norm to the usual $l^{\alpha}$-basis. Fix $n$. Then we may choose an operator $T$ : 
$B^{*} \rightarrow L^{1}$ with range contained in $\left[f_{1}, \ldots, f_{n}\right]$ so that $\|T\| \leqslant 2$, and so that there are norm-one elements $x_{1}, \ldots, x_{n}$ in $B^{*}$ with $T x_{i}=f_{i}$ for all $i$. Since $B$ is assumed to be $M$ - $p$-dominated, so is $B^{* *}$ by the local reflexivity principle. Hence $T^{*}$ is $p$-a.s. with $p$-a.s. norm at most $2 M$. Now Proposition 1.2 yields that

$$
\int\left(\sum\left|c_{i} f_{i}\right|^{q}\right)^{1 / q} d t \leqslant 2 M\left(\sum\left|c_{i}\right|^{q}\right)^{1 / q}
$$

for all scalars $c_{1}, \ldots, c_{n}$. We then obtain that for any sequence $\left(c_{i}\right)_{i=1}^{\infty}$ with $\sum\left|c_{i}\right|^{\alpha}<\infty, \sum\left|c_{i}\right|^{\alpha}\left|f_{i}\right|^{\alpha}<\infty$ almost everywhere. But this is false by a result of Schwartz [28] (see also Lemma 3 of [26]). This contradiction completes the proof of Theorem 2.1.

Fix $1<p<2$. A special case of Theorem 2.1 is the result of [24] that if $X$ is a subspace of $L^{1}$, then $I_{p}(X)=\infty$ if and only if $X$ is $p$-inclusive. It is possible to deduce this fact without using the special properties of stable random variables. Indeed, these properties are used only to prove the "if" assertion. Suppose $I_{p}(x) \neq \infty$ yet $X$ were $p$-inclusive. Then there would exist a subspace $Y$ of $L^{p}$, with the $p$ and 1-norms equivalent on $Y$, so that $Y$ is isomorphic to $X$. But then $Y$ would also be $p$-inclusive. It therefore follows by Proposition 2.3 that for all $n$ and $\lambda>1$, there exist norm-one elements $b_{1}, \ldots, b_{n}$ in $Y$ satisfying (7) for all scalars $c_{1}, \ldots, c_{n}$. A truncation lemma of P. Enflo and the author (Lemma 2.1 of [4]) now yields that fixing $0<\delta<1 / \lambda$ and $k$ a large number, then if $n$ is sufficiently large (e.g. so that $n^{1 / p-1 / 2}>-\frac{\lambda k}{1-\delta \lambda}$ ), there must exist an $i$ so that $\left(\int_{E_{i}} \mid b^{p}\right)^{1 / p}>\delta$, where $E=\left\{x:\left|b_{i}(x)\right|>k\right\}$. Since $\delta$ can be taken arbitrarily close to 1 , it easily follows that the $p$ and 1-norms on $Y$ are not equivalent, a contradiction.

We wish to conclude this section with a dual consequence of Theorem 2.1. Fix $2<p<\infty$, let $1 / p+1 / q=1$, and let $X$ be a Banach space. It follows easily from the remarks following the proof of 2.3 that if $X^{*}$ is $p$-inclusive, then $X^{* *}$ is $q$-inclusive. In fact, it follows from the local reflexivity principle that if some quotient-space of $X^{*}$ is $p$-inclusive, then $X$ itself is $q$-inclusive. On the other hand, if $X$ is $q$-inclusive, then $X^{*}$ has a $p$-inclusive quotient space. Indeed, a standard argument shows that if $X$ is $q$-inclusive, then every subspace of $X$ of finite codimension is also $q$-inclusive. The usual argument for producing finite-dimensional decompositions (f.d.d.'s) (as defined in [10]), and Proposition' 2.3 then show that there is a subspace $Y$ of $B$ having a f.d.d. $\left(Y_{n}\right)_{n=1}^{\infty}$ so that for each $n, Y_{n}$ is $n$-dimensional with a normalized basis $b_{1}^{n}, \ldots, b_{n}^{n}$ satisfying $\left(\sum\left|c_{i}\right|^{q}\right)^{1 / q} \leqslant 2\left\|\sum c_{i} b_{i}^{n}\right\|$ for all scalars $c_{1}, \ldots, c_{n}$. Thus there is a $\lambda$ so that each $Y_{n}$ is $\lambda$-complemented in $Y$, so $Y^{*}$ is $p$-inclusive. Theorem 2.1 then immediately gives:
COROLLARY 2.4. Let $p$ and $q$ be as above and let $B$ be an arbitrary Banach space. Then either $B$ is $q$-inclusive or every quotient space of $B^{*}$ is $p$-dominated. These alternatives are mutually exclusive.

A recent result of Maurey and Pisier [21], Exposé No. 8, asserts that if $r<2$ is such that $r=\sup \{s: B$ is of type-s $\}$, then $B$ is $r$-inclusive. Suppose $p$ and $q$ are as above and every quotient space of $B^{*}$ is $p$-dominated. Then (as pointed out to me by the above authors), $B$ is of type $q$; otherwise the Maurey-Pisier result yields that there is an $r \leqslant q$ so that $B$ is $r$-inclusive; but then of course $B$ is also $q$-inclusive, contradicting 2.4. On the other hand, results of Pisier ([24]; see also [21], Exposé No. 3) show that if $B$ is type- $q$, then $B$ is of type $q^{\prime}$-stable for all $q^{\prime}<q$, whence every quotient space of $B^{*}$ is $p^{\prime}$-dominated for all $p^{\prime}>p$. This cannot be improved to $B^{*}$ itself being $p$-dominated, for $L^{\alpha}$ is of type- $q$ but $\left(L^{q}\right)^{*}$ $=L^{p}$ is not $p$-dominated, being $p$-inclusive.

3. Unconditionally transitive bases and the Tzafriri function-space estimates. It is an open question if every Banach space of infinite dimension contains uniformly complemented $l_{n}^{p}$ s for $p=1,2$, or $\infty$. L. Tzafriri proved in [29] that this is indeed the case for Banach spaces $B$ with unconditional bases. Suppose $B$ is such a space. One of the key steps of his proof is to show the following: Suppose neither $B$ nor $B^{*}$ contains $l_{n}^{\infty}$ 's (which is the same, in general, as saying that $B$ does not contain uniformly complemented $l_{n}^{\infty}$ 's or $l_{n}^{1}$ 's). Then there exist a $2<p<\infty$ and a constant $K$ so that for all $n$, there exist $n$ terms $b_{1}, \ldots, b_{n}$ of the basis so that

$$
\frac{1}{K}\left(\frac{\sum\left|c_{i}\right|^{q}}{n}\right)^{1 / q} \leqslant\left(\frac{\left\|\sum c_{i} b_{i}\right\|}{\left\|\sum b_{i}\right\|}\right) \leqslant K\left(\frac{\sum\left|c_{i}\right|^{p}}{n}\right)^{1 / p}
$$

for all scalars $c_{1}, \ldots, c_{n}$ (where $1 / p+1 / q=1$ ). Inequalities (12) are called function-space estimates because they mean that the norm on the span of the $b_{i}$ 's is between the $L^{q}$ and $L^{p}$-norms, $L^{q}$ and $L^{p}$ being thought of as function spaces as opposed to the sequence spaces $l^{q}$ and $l^{p}$. Indeed, let $E_{1}, \ldots, E_{n}$ be the disjoint open intervals defined by $E_{i}=\left(\frac{i-1}{n}, \frac{i}{n}\right)$ for all $i$ and let $T$ be the map from the span of the $b_{i}$ 's into the simple functions on $[0,1]$ defined by $T\left(\sum c_{i} b_{i}\right)=L \sum c_{i} \chi_{E_{i}}$ for all scalars $c_{1}, \ldots, c_{n}$, where $L=\left\|\sum_{i=1}^{n} b_{i}\right\|$. Then inequalities (12) assert precisely that

$$
\frac{1}{K}\|T x\|_{q} \leqslant\|x\| \leqslant K\|T x\|_{p}
$$

for all $x$ in the span of the $b_{i}$ 's.

Tzafriri then concludes his argument as follows: suppose $n=2^{m}$. The span $R_{m}$ of the first $m$ Rademacher functions $r_{0}, \ldots, r_{m-1}$ is a sub- 
space of the range of $T$. Now the Khintchine inequalities yield that there is a constant $K_{p}$ depending only on $p$ so that $R_{m}$ is $K_{p}$-complemented in $L^{q}$ and $\|r\|_{p} \leqslant K_{p}\|r\|_{q}$ for all $r \in R_{m}$. It then follows that $T^{-1}\left(R_{m}\right)$ is $K_{p} K^{2}$-complemented in $\left[b_{1}, \ldots, b_{n}\right]$ and $K_{p}^{2} K^{2}$-isomorphic to $l_{m}^{2}\left(\left[b_{1}, \ldots, b_{n}\right]\right.$ denotes the linear span of $\left.b_{1}, \ldots, b_{n}\right)$. Since there is a $u$ independent of $n$ so that $\left[b_{1}, \ldots, b_{n}\right]$ is $u$-complemented in $B$, it follows finally that $B$ contains uniformly complemented $l_{m}^{2}$ 's. (Johnson and Trafriri [11] have I ecently considerably extended this result, by applying among others the techniques and results we have presented in the first two sections. - They prove that if a Banach lattice does not contain $l_{n}^{\infty}$ 's, then every infinite-dimensional subspace of the lattice contains uniformly complerented $l_{n}^{1}$ 's or $l_{n}^{2}$ 's.)

We shall show that a rather large class of finite unconditional bases ratisfy these function space estimates.

Defrnimions. Fix $n$ a positive integer and let $\left(b_{1}, \ldots, b_{n}\right)$ be a normalized basis in an $n$-dimensional Banach space $B$. We say that the basis is 1-U.-Tran. (for isometrically unconditionally transitive) if it is 1-unconditional and possesses a transitive group of permutations which extend to isometries of the space. That is, there exists a group $\mathscr{D}$ of permutations of $\{1, \ldots, n\}$ so that for all $1 \leqslant i, j \leqslant n$ there is a $\sigma \in \mathscr{D}$ with $\sigma(i)=j$, so that for each $\sigma \in \mathscr{D}$, the linear operator $g_{\sigma}$ on $B$ defined by $g_{\sigma}\left(b_{i}\right)=b_{\sigma(i)}$ for all $i$, is an isometry on $B$. In other words, for all choices of signs \pm , all $\sigma \in \mathscr{D}$, and all scalars $c_{1}, \ldots, c_{n}$,

$$
\left\|\sum_{i=1}^{n} \pm c_{i} b_{\sigma(i)}\right\|=\left\|\sum c_{i} b_{i}\right\|
$$

We put $G=\left\{g_{\sigma}: \sigma \epsilon \mathscr{D}\right\}$. Now let $1<\lambda$; a normalized basis $\left(b_{1}, \ldots, b_{n}\right)$ in $B$ is said to be $\lambda$-U.-Tran. if it is $\lambda$-equivalent to a 1-U.-Tran. basis $\left(b_{1}^{\prime}, \ldots, b_{n}^{\prime}\right)$ in some $B^{\prime}$; i.e., if the map $T: B \rightarrow B^{\prime}$ so that $T b_{i}=b_{i}^{\prime}$ for all $i$ satisfies $\|T\|\left\|T^{-1}\right\| \leqslant \lambda$.

Hence are some examples of such phenomena. Let $\tau \geqslant 1$; say that a normalized basis is $\tau$-stationary (resp. $\tau$-spreading) if for all scalars $c_{1}, \ldots$ $\ldots, c_{n}$ and all $k$ with $1 \leqslant k \leqslant n,\left\|\sum_{i=1}^{k_{k}} c_{i} b_{i}\right\| \leqslant \tau\left\|\sum_{i=1}^{k_{k}} c_{i} b_{i+j}\right\|$ for all $j \leqslant n-k$ (resp. $\left\|\sum_{i=1}^{k} c_{i} b_{i}\right\| \leqslant \tau\left\|\sum_{i=1}^{k} c_{i} b_{m_{i}}\right\|$ for all $m_{1}<m_{2}<\ldots<m_{l} \leqslant n$ ). (Evidently a $\tau$-spreading basis is automatically $\tau$-stationary.) Then every $\tau$-stationary $u$-unconditional basis is $2 u \tau$-U.-Tran. Indeed, for a $\tau$-stationary $u$-unconditional basis $\left(b_{1}, \ldots, b_{n}\right)$, let $\mathscr{D}$ be the transitive group of permutations, $\mathscr{D}=\left\{\sigma_{1}, \ldots, \sigma_{n}\right\}$, so that for each $i, 1 \leqslant i \leqslant n, \sigma_{i}$ shifts $1, \ldots, n-i+$ +1 to $i, i+1, \ldots, n$ and $n-i+2, \ldots, n$ to $1, \ldots, i-1 ;$ i.e., $\sigma_{i}(j)=j+i-1$ for $1 \leqslant j \leqslant n-i+1$ and $\sigma_{i}(j)=j+i-n-1$ for $n-i+1<j \leqslant n$. Now define a new norm $\|\cdot\| \cdot \|$ on $B$ by $\left\|\sum c_{i} b_{i}\right\|=\sup _{ \pm g \in \mathscr{D}}\left\|\sum \pm c_{i} b_{g(i)}\right\|$ for all scalars $c_{1}, \ldots, c_{n}$. Then $\left(b_{1}, \ldots, b_{n}\right)$ forms a 1-U.-Tran. basis for $B$ endowed with ||$\cdot \mid \|$, and it is not difficult to see that the two norms are $2 u \tau$-equivalent. It seems likely that there exist U.-Tran. bases which are not stationary; precisely,

CONJECTURE. Fror every $\tau>1$ there exists a 1-U.-Tran. basis which has no $\tau$-stationary permutation.

(P. Wojtaszczyk has recently shown that for every $\tau>1$ there exists a 1-stationary 1-unconditional basis which is not $\tau$-spreading.)

In order to develop inequalities (12) for suitable U.-Tran. bases, we first need the following basic fact.

LEIMMA 3.1. Let $\left(b_{1}, \ldots, b_{n}\right)$ be a 1-U.-Tran. basis with biorthogonal functionals $b_{1}^{*}, \ldots, b_{n}^{*}$. Then

$$
\left\|\sum_{i=1}^{n} b_{i}\right\| .\left\|\sum_{i=1}^{n} b_{1}^{*}\right\|=n .
$$

Proof. We have that $\left\|\Sigma b_{i}^{*}\right\|=\sup \left|\sum b_{i}^{*}(x)\right|$, the supremum taken over all $x=\sum c_{i} b_{i}$ of norm one. But fix such an $x$; then $\left|\sum b_{i}^{*}(x)\right|=\left|\sum c_{i}\right|$ $\leqslant \sum\left|c_{i}\right|=\sum b_{i}^{*}(|x|)$, where we set $|x|=\sum\left|c_{i}\right| b_{i}$. Since the basis is 1-unconditional, $\||x|\|=1$ also. We thus have that $\left\|\sum b_{i}^{*}\right\|=\sup \frac{\sum c_{i}}{\left\|\sum c_{i} b_{i}\right\|}$ the supremum over all choices of $c_{i}$ 's with $\sum c_{i}>0$ and $c_{i} \geqslant 0$ for all $i$. But then by homogeneity we may restrict our attention to $c_{i}$ 's with $\sum c_{i}=1$ and $c_{i} \geqslant 0$ for all $i$. Now let $C$ denote the convex hull of the $b_{i}$ 's; $C$ is of course closed and we have shown that $\left\|\sum b_{i}^{*}\right\|^{-1}=\inf _{x \in O}\|x\|$. Naturally, this infimum is achieved for some $x \in C$. Let $G$ and $\mathscr{D}$ be chosen as in the definition of a 1-U.-Tran. basis. Now since the set $\left\{b_{1}, \ldots, b_{n}\right\}$ is invariant under the action of $G$, so is $C$.

Thus, letting $k$ equal the number of elements in $G$, we have that $y=\frac{1}{k} \sum_{g \in G^{*}} g(x)$ also belongs to $G$, being a convex combination of elements of $C$, and, moreover, $\|y\|=\left\|\sum b_{i}^{*}\right\|^{-1}$. But we also have that $g(y)=y$ for all $g \in G$, since $\mathscr{D}$ is transitive; this implies that $y=\frac{1}{n} \sum_{i=1}^{n} b_{i}$ which proves 3.1. (Of course, the last part of the argument is just a very elementary version of the Kakutani fixed-point theorem.)

We are now prepared for the main result of this section.

Proposimion 3.2. Let $2 \leqslant p<\infty, 1 / p+1 / q=1, \lambda, M \geqslant 1, n$ a positive integer, and $\left(b_{1}, ., \ldots, b_{n}\right), a \lambda-U$.-Tran. basis for the Banach space $B$. Then 
if $B$ is $M-p$-dominated,

$$
\frac{1}{\lambda} \frac{\sum\left|c_{i}\right|}{n} \leqslant \frac{\left\|\sum c_{i} b_{i}\right\|}{\| \sum b_{i} \mid} \leqslant \lambda^{2} M\left(\frac{\sum\left|c_{i}\right|^{\beta}}{n}\right)^{1 / p}
$$

for all scalars $c_{1}, \ldots, c_{n}$.

If $B^{*}$ is $M$-p-dominated,

(14) $\frac{1}{\lambda^{2} M}\left(\frac{\sum\left|c_{i}\right|^{q}}{n}\right)^{1 / a} \leqslant \frac{\left\|\sum c_{i} b_{i}\right\|}{\left\|\sum b_{i}\right\|}$ for all scalars $c_{1}, \ldots, c_{n}$.

Consequently, if both $B$ and $B^{*}$ are $M$-p-dominated, then the basis $\left(b_{1}, \ldots, b_{n}\right)$ satisfies the Tafriri function-space-estimates (12) for $K=\lambda^{2} M$.

Remark. Inequalities (13) also yield that for $n=2^{m}$, the Rademacher functions over the basis span a $\beta$-isomorph of $l_{m}^{2}$, where $\beta$ depends only on $M, P$, and $\lambda$.

Proof of 3.2. It is easily seen that the general case follows from the $\lambda=1$-case, so we assume $\lambda=1$. (The $\lambda^{2}$ term arises because one first requires the evident fact that if $B$ is $\lambda$-isomorphic to $B^{\prime}$ and $B$ is $M-p$ dominated, then $B^{\prime}$ is $\lambda M$ - $p$-dominated.) Now (14) follows from (13) and Lemma 3.1. Indeed, once (13) is established we obviously have that $\left(b_{1}^{*}, \ldots, b_{n}^{*}\right)$ is a 1-U.-Tran. basis for $B^{*}$, where $b_{1}^{*}, \ldots, b_{n}^{*}$ are the biorthogonal functionals to $b_{1}, \ldots, b_{n}$. Thus

$$
\frac{\left\|\sum c_{i} b_{i}^{*}\right\|}{\left\|\sum b_{i}^{*}\right\|} \leqslant M\left(\frac{\sum\left|c_{i}\right|^{p}}{n}\right)^{1 / p} \quad \text { for all scalars } c_{1}, \ldots, c_{n} .
$$

But then taking the adjoint of the natural map from $l_{n}^{p}$ into $B^{*}$, we have that for all such scalars,

$$
\left(\sum\left|c_{i}\right|^{q}\right)^{1 / \alpha} \leqslant \frac{M}{n^{1 / p}}\left\|\sum b_{i}^{*}\right\|\left\|\sum c_{i} b_{i}\right\| .
$$

(14) follows immediately upon applying the equality of Lemma 3.1. The first inequality in (13) also follows directly from Lemma 3.1 and the fact that $b_{1}, \ldots, b_{n}$ is 1 -unconditional. It remains to prove the second inequality in (13). Since $\left\|\Sigma b_{i}\right\|=\left\|\Sigma \pm b_{i}\right\|$ for all choices of \pm , it follows from inequality (4) of Section 1 and the definition of " $M$-p-dominated" that there exist positive numbers $\mu_{1}, \ldots, \mu_{n}$ with $\sum \mu_{i}=1$ so that

$$
\left\|\sum c_{i} b_{i}\right\| \leqslant M\left\|\sum b_{i}\right\|\left(\sum\left|c_{i}\right|^{p} \mu_{i}\right)^{1 / p}
$$

or all scalars $c_{1}, \ldots, c_{n}$.

Now let $\mathscr{D}$ be as in the definition of a 1-U.-Tran. basis, fix the scalars $c_{1}, \ldots, c_{n}$, and let $\sigma \in \mathscr{D}$. Then $\left\|\sum c_{i} b_{i}\right\|=\left\|\sum c_{i} b_{\sigma(i)}\right\|=\left\|\sum c_{\sigma}-\mathbf{1}(i) b_{i}\right\|$ and $\sum\left|c_{\sigma^{-1}(i)}\right|^{p} \mu_{i}=\sum\left|c_{i}\right|^{p} \mu_{\sigma(i)}$, the first equality holding by the definition of $\mathscr{D}$; the second two equalities are trivial. These facts and (15) yield

$$
a \leqslant \sum_{i=1}^{n}\left|c_{i}\right|^{p} \mu_{\sigma(i)}, \quad \text { where } \quad a=\left\|\sum c_{i} b_{i}\right\|^{p} \mid M^{p}\left\|\sum b_{i}\right\| .
$$

Finally, letting $k$ equal the number of elements of $\mathscr{D}$, we have that

$$
a \leqslant \sum_{i=1}^{n}\left|c_{i}\right|^{p}\left(\frac{1}{\hbar} \sum_{\sigma \in \mathscr{D}} \mu_{\sigma(i)}\right)
$$

Since $\mathscr{D}$ is transitive, we have that for all $i, \frac{1}{k} \sum_{\sigma \in \mathscr{D}} \mu_{\sigma(i)}=\frac{1}{k} \sum_{\sigma \in \mathscr{D}} \mu_{\sigma(1)}=\frac{1}{n}$. Thus; $a \leqslant \frac{1}{n} \sum_{i=1}^{n}\left|c_{i}\right|^{p} ;$ since $c_{1}, \ldots, c_{n}$ are arbitrary scalars (13) is proved.

We wish to make some final comments concerning Tzafriri's results. Suppose that $\left(u_{j}\right)_{j=1}^{\infty}$ is a normalized unconditional basis for a space $B$, so that neither $B$ nor $B^{*}$ contains $l_{n}^{\infty}$ 's. Then by Theorem $1^{\prime}$, there exists a $2<p<\infty$ so that both $B$ and $B^{*}$ are $p$-dominated. Because of the unconditionality of the basis, we have that there is a constant $M$ so that for any $n$ and $n$ terms of the basis $b_{1}, \ldots, b_{n}$, both $\left[b_{1}, \ldots, b_{n}\right]$ and $\left[b_{1}, \ldots, b_{n}\right]^{*}$ are $M$-p-dominated. Now as pointed out by Tzafriri, Ramsey's theorem implies that there is a $\tau$ so that for all $n$, there are $n$ terms of the basis $b_{1}, \ldots, b_{n}$ so that $\left(b_{1}, \ldots, b_{n}\right)$ is $x$-spreading. But then there is a $\lambda$ so that for all such $b_{1}, \ldots, b_{n},\left(b_{1}, \ldots, b_{n}\right)$ is $\lambda$-U.-Tran. Consequently, Proposition 3.2 and the Khintchine inequalities yield a nicely complemented $l_{m}^{2}$ (where $m \sim \log n$ ) inside $\left[b_{1}, \ldots, b_{n}\right]$, which is in turn nicely complemented in the whole space due to the unconditionality of the basis.

The relevance of Ramsey's theorem to Banach space theory was originally discovered by Brunel and Sucheston (see [1]). As noted in [29], their arguments together with Tzafriri's results provide a combinatorialanalytical proof that every infinite-dimensional Banach space contains $l_{n}^{2}$, s uniformly. A recent remarkable discovery of Krivine [13] implies that if a Banach space contains $l_{n}^{p}$ 's uniformly (for some $1<p<\infty$ ), that is, if there is a $K$ so that it contains a $K$-isomorph of $l_{n}^{p}$ for all $n$, then it contains a $\lambda$-isomorph of $l_{n}^{p}$ for all $\lambda>1$ and $n$. Since Krivine's arguments are also combinatorial-analytical, an alternate proof of Dvoretzky's theorem is now available. As pointed out to me by B. Maurey, recent results due jointly to himself and Pisier [19], together with Krivine's discovery, yield the following: Let $B$ be a given Banach space, and let $p=\sup \{q \geqslant 2$ : $B$ is $q$-inclusive $\}$ or $p=\inf \{q \leqslant 2: B$ is $q$-inclusive $\}$. Then $B$ contains a $\lambda$-isomorph of $l_{n}^{p}$ for all $\lambda>1$ and $n$. 


\section{References}

[1] A. Brunel and L. Sucheston, On B-convex Banach spaces, Math. Systems Th. 7 (1974), pp. 294-299.

[2] E. Dubinski, A. Pełczyński and H. P. Rosenthal, On Banach spaces $X$ for which $\Pi_{2}\left(\mathscr{L}_{\infty}, X\right)=B\left(\mathscr{L}_{\infty}, X\right)$, Studia Math. 44 (1972), pp. 617-648.

[3] A. Drorotzky, Some results on convex bodies and Banach spaces, Proc. International Symp. on Linear Spaces, Jerusalem Ac. Press LTD, Jerusalem 1961 pp. $123-160$.

[4] P. Enflo and H. P. Rosenthal, Some results concerning $L^{p}(\mu)$-spaces, J. of Funct. Anal. 14 (1973), pp. 325-348.

[5] T. Figiel and W. B. Johnson, A uniformly convex Banach space which contains no $l_{p}$, to appear.

[6]. D. P. Giesy, On a convexity condition in normed linear spaces, Trans. Amer. Math. Soc. 125 (1966), pp. 114-146.

[7] Y. Gord on and D. Lewis, Banach ideals on Hilbert spaces (to appear).

[8] A. Grothendieck, Résumié de la théorie metrique des produits tensoriels topologiques, Bol. Soc. Mathem., Sao Paulo 8 (1956), pp. 1-79.

[9] W. B. Johnson, On finite dimensional subspaces of Banach spaces with local unconditional structure, Studia Math. 51 (1974), pp. 223-238.

[10] -, H. P. Rosenthal, and M. Zippin, On bases, finite-dimensional decompositions and weaker structures in Banach spases, Israel J. Math. 9 (1971), pp. 488-506.

[11] - and L. Tzafriri, On the local structure of subspaces of Banach lattices, Israel J. Math. 20 (1975), pp. 292-299.

[12] J.-P. K ahane, Some random series of functions, D. C. Heath and Co. Lexington, Mass. 1968.

[13] J. L. Krivine, Sur les espaces isomorphes a $l^{p}$, to appear.

[14] S. KWapień, On a theorem of $L$. Schwartz and its applications to absolutely summing operators, Studia Math. 38 (1970), pp 193-201.

[15] J. Lindenstrauss and A. Pelczyński, Absolutely summing operators in $\mathscr{L}_{p^{-}}$ spaces and their applications, ibid. 29 (1968), pp. 275-326.

[16] J. Lindenstrauss and H. P. Rosenthal, The $\mathscr{L}_{p^{-s p a c e s}}$, Israel J. Math. 7 (1969), pp. 325-349.

[17] J. Lindenstrauss and L. Tzafriri, Olassical Banach spaces, Lecture note in Mathematics 338, Springer-Vorlag 1973.

[18] B. Maurey, Théoremes de factorisation pour les opérateurs linéaires à valeurs dans les espaces $L^{p}$, Société Mathématique de France, Astérisque no 11 (1974), pp. 1-163.

[19] B. Maurey and G. Pisier Séries de variables aléatoires vectorielles indépendantes et propriétés géométriques des espaces de Banach, Studia Math. this vol., pp. 49-90.

[20] - and L. Schwartiz, Espaces $L^{p}$ et applications raidonifiantes, Seminaire Maurey-Schwartz, Ecole Polytechnique, Paris 1972-1973.

[21] - - Espaces $L^{p}$, applications radonifiantes et géométrio des espaces do Banaoh, Seminaire Maurey-Schwartz, Ĺcole Polytechnique, Paris 1973-1974.

[22] A. Persson and A. Piets ch, $p$-nulleare und p-integrale Abbildungen in Banachräumen, Studia Math. 33 (1969), pp. 19-62.

[23] A. Pietsch, Absolute p-summierende Abbildungen in normirten Räumen, ibidem 28 (1967), pp. 333-353.

[24] G. Pisier, Type des espaces normés, C. R, Acad. Se. Paris 276 (1973), pp. 16731976 .
[25] H. P. Rosenthal, On the span in $L^{p}$ of sequences of independent random variables $\Pi$, Proc. 6th Berkeley Symp. on Mathematical Stat. and Prob. Vol. II (1972), pp. 149-167.

[26] - On subspaces of Lp, Annals of Math. 97 (1973), pp. 344-373.

[27] - The Banach spaces $O(K)$ and $L^{p}(\mu)$, Bull. Amer. Math. Soc. 81 (1975), pp. $763-782$

[28] L. Schwartz, Applications radonifiantes, Seminaire d'Analyse de l'École Polytechnique, Paris 1969-1970.

[29] L. Tzafriri, On Banach spaces with unconditional bases, Israel J. Mat. 17 (1974), pp. 84-93.

UNIVIRRITY OF ILLINOIS AT URBANA-CHAMPAIGN 